\title{
Analytical study of frame aggregation level to infer IEEE 802.11 network load
}

\author{
Nour El Houda Bouzouita \\ Univ de Lyon, UCB Lyon 1 \\ Inria, ENS de Lyon, CNRS \\ LIP UMR 5668, \\ Lyon, France
}

nour-el-houda.bouzouita@ens-lyon.fr

\author{
Anthony Busson \\ Univ de Lyon, UCB Lyon 1 \\ Inria, ENS de Lyon, CNRS \\ LIP UMR 5668, \\ Lyon, France \\ anthony.busson@ens-lyon.fr
}

\author{
Herve Rivano \\ Univ Lyon, INSA Lyon \\ Inria, CITI \\ CITI EA 3720, \\ Lyon, France \\ herve.rivano@inria.fr
}

\begin{abstract}
Over the past two decades, Wi-Fi technology (defined by the IEEE 802.11 standard) has become a prominent wireless network access technology. In many situations, a device may attach to several Wi-Fi access points within the radio range. The operating system makes its choice over metrics that do not take into account the actual available capacity. To fill this gap, several proposals have been made to infer capacity, for example by estimating the occupied proportion of the channel. However, these techniques are being thwarted by the mechanisms recently introduced in $\mathbf{8 0 2 . 1 1}$ to improve transmission speeds, in particular frame aggregation. In this article, we focus on busy time inference based on frame aggregation level. We propose an analytical model based on a Markov chain which estimates the theoretical aggregation level for different amounts of cross traffic. We validate its accuracy against simulations carried out on the ns-3 network simulator and an ad-hoc simulator. Results show that the theoretical model gives an accurate estimation of the frame aggregation level and that it can be used to infer the network load.
\end{abstract}

Index Terms-IEEE 802.11, Frame aggregation, A-MPDU, Markov model

\section{INTRODUCTION}

WLANs (Wireless Local Area Networks) have become ubiquitous in our daily lives. IEEE 802.11, also known as Wi$\mathrm{Fi}$, is the most popular wireless access network technology. This technology is offered almost everywhere, in companies, home and public areas [1].

On the one hand, companies and universities may deploy a set of Access Points (AP) corresponding to the same logical Wi-Fi network (Extended Service Set) and identified by a common Service Set ID to cover properly the users. An Extended service set is managed by a centralized server that applies algorithms (e.g., load balancing, the optimal channel allocation, security algorithms, etc.) to improve performance and enable a flexible management of the network resources [2]. On the other hand, the spatial densification of Wi-Fi networks is also related to the fact that many different Wi-Fi networks can be deployed in the same area. Different servers manage these APs and there is no common policy to help the devices selecting the Wi-Fi network that offers the best performance.

This problem and the need to enhance the overall performance of the network have led prior works to estimate the available bandwidth which is defined as the maximum unused bandwidth at a link or path as it directly impacts application performance and user quality of experience. Some applications such as iperf and speedtest (e.g., Ookla Speedtest [3]) consume all the available bandwidth to estimate the throughput. Despite their accuracy, it is preferable to avoid their intrusiveness. We therefore consider only approaches that introduce a small measurement overhead to infer the available bandwidth. Besides, the available bandwidth is specific to a device, its channel and antenna gains and many other hardware or software parameters that condition the modulation and coding scheme it can use. A more versatile metric is the busy time estimation, defined as the proportion of time the wireless medium is busy. It captures concurrent transmissions, constituting the AP load, as well as inter-networks interference. This estimation allows a device to identify the AP that offers the highest availability.

In the most recent evolutions of 802.11, in particular $802.11 \mathrm{n}$ or ac, a frame aggregation mechanism has been introduced. In this paper, we study the possibility to infer the channel busy time from the frame aggregation level measured for a probe traffic. We propose two Markov chain based analytical models to estimate the theoretical aggregation level of a probe traffic (generated by the busy time estimation tool) concurrent to a cross traffic (the current load). The first is considering a cross traffic that also uses frame aggregation, while the second captures cross traffic without aggregation. Our model is compared to a custom-made simulator and ns-3 [18] simulations, both allowing more generic traffic patterns and realistic scenarios. For the considered scenario, we show that the mean aggregation level can be an accurate metric to estimate the load of an AP.

The rest of this paper is organized as follows. Section II summarizes related works. Section III briefly describes the frame aggregation scheme introduced since IEEE 802.11n. Our analytical models are presented in section IV. Numerical results are presented in Section V. We discuss the possibility to use the aggregation level to estimate the busy time in Section VI. Finally, Section VII concludes this paper.

\section{RELATED WORK AND MOTIVATION}

Non-intrusive available bandwidth estimation techniques have first been developed for wired networks. They can be 
classified into two categories: Packet Rate Model (PRM) and Probe Gap Model (PGM). PRM techniques are based on comparing the emission and reception rates of small probe packets. Techniques such as Train of packet pairs (TOPP) [13], pathload [4], pathChirp [14] and DietTOPP [12], inject an increasing probe traffic in the network. The concurrency with other packets, also denoted cross traffic, will impact the reception rates. These methods differ according to the probing rate generation.

Alternatively, PGM techniques leverage the waiting times induced by Carrier Sense Multiple Access (CSMA) to estimate the cross traffic. Spruce [8] and Initial Gap Increase/Packet Transmission Rate (IGI/PTR) [16] send batches of probe packets and measure the inter-arrival time of the consecutive packets to infer the available bandwidth.

More recent works have also taken into account the 3G/4G networks [20] and the cloud networks [19]. However, in this paper we focus on Wi-Fi networks.

a) Available bandwidth estimation in IEEE 802.11 networks: The waiting times induced by the CSMA/CA of 802.11 are different and more complex than those of Ethernet and wired networks. To cope with it and address Wi-Fi networks, more complex schemes have been developed. IdleGap [15] needs to run on a real-time system and infers the idle time from a low layer information, making it difficult to implement in practice. WBest [5] is a PGM technique composed of two stages. It first sends pairs of probe packets and estimates the capacity of the network. It then transmits a train of probe packets at the consequent rate and deduces the available bandwidth. Unfortunately, these schemes fail when the frame aggregation mechanism implemented in the recent 802.11 amendments is enabled. Indeed at the application layer, aggregated frames are received simultaneously. PGM techniques cannot process null inter-arrival times and PRM techniques may misinterpret and overestimate the reception rate.

b) Aggregation aware available bandwidth estimation: WBest+ [6] is an enhanced version of WBest that considers aggregated frames as jumbo frames. The available bandwidth estimation considers the time between aggregated frames instead of the time between probe packets. AIWC [7] estimates the frame aggregation level at the receiver to measure link congestion and deduce the available bandwidth. To detect an aggregation, both WBest+ and AIWC follow a threshold-based method: if an inter-arrival time is below a given threshold, the two packets are considered as aggregated. The parameterization of these methods is quite empirical. A formal study of the aggregation behavior as a function of the traffic has to be performed to settle these kinds of techniques.

c) Frame aggregation analysis: Markovian models capturing frame aggregation have been introduced under saturated conditions [10], [11]. A Discrete Time Markov Chain (DTMC) has been proposed to study the frame aggregation post-backoff and evaluate the throughput performance under unsaturated conditions [9]. The scenarios considered in this paper are however not suitable for available bandwidth evaluation.

In this paper, we model and simulate scenarios in which a

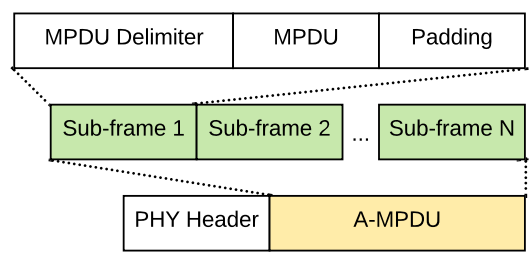

Fig. 1: A-MPDU frame aggregation.

device estimates the busy time on a Wi-Fi network with frame aggregation enabled. We consider two kinds of traffic sharing the medium: a cross traffic corresponding to the network usage that can aggregate frames or not and a deterministic probe traffic. We want to study the relationship between channel busy time and frame aggregation level to determine if the latter can characterize the channel load.

\section{802.11 FRAME AGGREGATION}

IEEE 802.11n amendment made several changes to the MAC layer to improve network efficiency and channel utilization. In this section, we briefly describe one of the most important MAC enhancement which is the frame aggregation. Frame Aggregation scheme aims to increase Wi-Fi throughput by sending multiple data packets in a single transmission using a larger aggregated data frame to reduce the MAC layer overhead. IEEE $802.11 \mathrm{n}$ amendment, and the most recent IEEE 802.11 standard [17] have defined two types of frame aggregation: Aggregate MAC Protocol Data Unit (A-MPDU) and Aggregate MAC Service Data Unit (A-MSDU). A-MSDU is an aggregation of several SDUs (Service Data Units) with one common MAC header. It is rarely implemented and we do not consider it in this article.

On the contrary, A-MDPU aggregation is enabled by default in recent Wi-Fi cards. It consists in sending multiple MPDU sub-frames to the same receiver with a common PHY header. Each sub-frame keeps its own 802.11 MAC header and a frame check sequence (FCS), starts with a MPDU delimiter and ends with padding bytes. Therefore, the corruption of a single MPDU sub-frame does not require the retransmission of the whole A-MPDU frame. The structure of an aggregated frame is shown in Fig. 1.

The frame aggregation level is thus dependent on the buffer state and the network load, in particular the time to access the medium.

\section{MODEL DESCRIPTION}

In order to infer the behavior of frame aggregation in congested and non-congested networks, we propose an analytical model. It estimates the aggregation distribution defined as the probability for a frame to aggregate $n$ sub-frames using the AMPDU mechanism for a given busy time fraction. The model is based on a Markov chain. 
a) First model: Cross traffic does aggregate: Our system is a wireless network using the IEEE 802.11 Distributed Coordination Function (DCF) to access the radio channel. Two traffics share the capacity: a cross traffic and a probe traffic.

- Probe traffic is sent by the busy time estimation tool from a client to a server via the wireless link with a specified data rate. The destination uses the aggregation level of this probe traffic to estimate the network load The proposed Markov chain estimates the aggregation distribution of this probe traffic. It is a constant bit rate (CBR) traffic where packets are generated at regular interval $d_{p}$ by the application. We assume that the buffer has a maximum size $K_{\max }$. It is also the maximum size of the aggregated frames. Consequently, the buffer becomes empty each time a probe traffic frame is sent. The random process describing the number of aggregated sub-frames contained in the $n^{\text {th }}$ transmitted frame for this probe traffic is denoted $X_{n}$. It takes its values in the set $\left\{0, \ldots, K_{\max }\right\}$.

- Cross traffic represents the concurrent traffic between other devices and the AP in the radio range of the node that uses the busy time estimation tool. In our Markov chain, this cross traffic is modeled through a unique traffic sent over the channel and managed by a unique queue. It is also a CBR traffic generated at regular interval $d_{c}$. $Y_{n}$ is the random process that describes the number of packets in the cross traffic buffer at the moment of the $n^{t h}$ probe frame transmission (that contains $X_{n}$ sub-frames). The frame aggregation mechanism is the same as the probe traffic. The buffer has a maximum size of $K_{\max }$. It is emptied when an aggregated frame is sent. The possible states are in $\left\{0, \ldots, K_{\max }\right\}$.

There is a strong dependency between the processes $X_{n}$ and $Y_{n}$. When an aggregated frame is sent, its length impacts the transmission duration and consequently the number of packets received in the probe and cross traffic buffers. The two processes have to be considered conjointly. The Markov chain is thus defined as the couple $\left(X_{n}, Y_{n}\right)_{n \geq 0}$. It is assumed homogeneous.

We consider the transition probability $P_{(i, j)(l, m)}$ from state $(l, m)$ to state $(i, j)$ defined as:

$$
P_{(i, j)(l, m)}=\mathbb{P}\left(\left(X_{n+1}, Y_{n+1}\right)=(i, j) \mid\left(X_{n}, Y_{n}\right)=(l, m)\right)
$$

The transition probabilities are fully determined by the time between two consecutive probe traffic transmissions. As both probe and cross traffics are deterministic, this time sets the number of packets that arrived in the two buffers between two transmissions and thus the number of frames that will be sent in the aggregated frame.

Consequently, we analyze the events that may occur between two probe traffic transmissions. Fig. 2 shows an example of the possible events between two probe transmissions. Let assume that the current state of the Markov chain at step $n$ is $(l, m)$, i.e. $\left(X_{n}=l, Y_{n}=m\right)$. First, the probe traffic frame is sent. The transmission duration is denoted

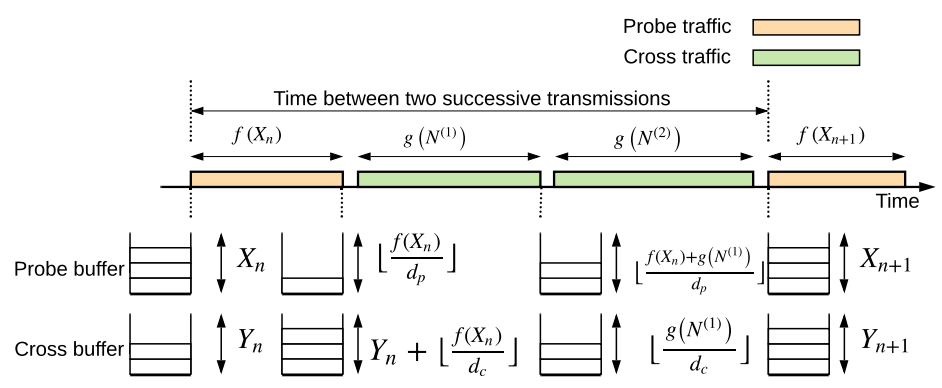

Fig. 2: Possible events between two successive probe transmissions. At the step $n$, the $n^{\text {th }}$ probe frame is transmitted. It contains $X_{n}$ sub-frames. Its duration is $f\left(X_{n}\right)$. The competing station accesses to the medium to transmit the previous data at its buffer $\left(Y_{n}\right)$ plus the packets that arrived during the period $f\left(X_{n}\right)$. the amount of time to send those packets is given by $g\left(\left\lfloor Y_{n}+\frac{f\left(X_{n}\right)}{d c}\right\rfloor\right)$. In this case, between the transmission of the $X_{n}$ and the $X_{n+1}$ frames, the cross traffic succeeds to access the medium two successive times.

$f(l)$. Note that if $l>1$, it is an A-MPDU frame that contains $l$ aggregated packets. $f(l)$ counts the time to access the medium (composed of the Distributed Inter-Frame Space (DIFS), and the mean backoff estimated as $\frac{C W m i n}{2}$. SlotTime where CWmin denotes the minimum contention window size), the physical header (PHY Overhead), the MAC header, the payload and the Frame Check Sequence (FCS), the shortest Inter-frame Space (SIFS), and the Ack or BlockAck. We get:

$$
\begin{aligned}
f(l)= & \text { DIFS }+\frac{C W \text { min }}{2} \cdot \text { SlotTime } \\
& + \text { PHYOverhead }+ \text { SIFS }+ \text { BlockACK } \\
& +\frac{(\text { MacHeader }+ \text { PacketSize }+ \text { FCS }) \times 8 \times l}{\text { Physical transmission rate }}
\end{aligned}
$$

During this transmission, the number of received packets that arrived in the two buffers can be approximated by $\left\lfloor\frac{f(l)}{d_{p}}\right\rfloor$ and $\left\lfloor\frac{f(l)}{d_{c}}\right\rfloor$ for probe and cross traffic respectively. At the end of this transmission, the probe traffic buffer contains $\left\lfloor\frac{f(l)}{d_{p}}\right\rfloor$ packets, and the cross traffic buffer contains $N^{(1)}=m+\left\lfloor\frac{f(l)}{d_{c}}\right\rfloor$ packets.

Before the next probe transmission, several successive transmissions of cross traffic may occur. Let $N^{(k)}$ be the number of packets in the cross traffic buffer at the time when the cross traffic tries to access the medium for the $k^{t h}$ time. $N^{(1)}$ has already been computed and corresponds to the buffer size at the end of the probe traffic transmission. If it succeeds to access the medium (assuming that $N^{(1)}>0$ ), a frame or aggregated frame composed of $N^{(1)}$ packets is sent. During this transmission, $N^{(2)}$ packets arrived in the cross traffic buffer with: 


$$
N^{(2)}=\left\lfloor\frac{g\left(N^{(1)}\right)}{d_{c}}\right\rfloor
$$

The function $g(x)$ is the duration of the transmission of a frame $(x=1)$ or an aggregated frame (with $x>1$ sub-frames). The only difference with $f($.$) is the physical$ transmission rate and the packet size that can be different from the probe traffic.

More generally, for $k>1$, we get:

$$
N^{(k)}=\left\lfloor\frac{g\left(N^{(k-1)}\right)}{d_{c}}\right\rfloor
$$

Now, we compute the probability that $k$ cross traffic frames are sent successively. It is denoted $\mathbb{P}(Q(l, m)=k)$ where $m$ and $l$ denote the buffer states as in the previous equations and $Q(l, m)$ is the number of successive times that the cross traffic accesses to the medium. $k=0$ means that the cross traffic does not access to the medium between two successive probe traffic transmissions. It can be due to an empty buffer or because the probe traffic wins access to the medium. We denote $p(k)$ the probability for the cross traffic to access the medium $k$ successively times given that probe and cross traffics have non-empty buffers. This probability depends on the contention window and $k$. We get:

$\mathbb{P}(Q(l, m)=0)=\mathbb{1}_{m+\frac{f(l)}{d c}<1}+(1-p(1)) \cdot \mathbb{1}_{m+\frac{f(l)}{d c} \geq 1}$

For $k>0$, we get,

$$
\begin{array}{r}
\mathbb{P}(Q(l, m)=k)=p(k) \cdot \prod_{q=1}^{k} \mathbb{1}_{N^{(q)} \geq 1} \cdot\left(\mathbb{1}_{N^{(k+1)}=0}\right. \\
\left.+(1-p(k+1)) \mathbb{1}_{N^{(k+1)}>0}\right)
\end{array}
$$

In this equation, the product corresponds to the probability that the cross traffic has a non-empty buffer during each of the $k$ successive transmissions. The last term is the probability that the cross traffic does not access to the medium after its $k^{\text {th }}$ transmission either because it loses when competing with the probe traffic or because of an empty buffer.

To obtain the transition probabilities we condition by the number of cross traffic accesses and their transmission times. As the probe traffic is $\mathrm{CBR}$, the number of frames in the probe traffic buffer is directly deduced from this time. For $i \geq 2$ we obtain:

$$
\begin{aligned}
P_{(i, j)(l, m)}= & \sum_{\substack{k=0\\
}}^{\infty} \mathbb{P}(Q(l, m)=k) \\
& \cdot \mathbb{1}_{d_{p} \cdot i \leq f(l)+\sum_{q=1}^{k} g\left(N^{(q)}\right)<d_{p} \cdot(i+1)} \cdot \mathbb{1}_{N^{(k+1)}=j}
\end{aligned}
$$

For $i=1$ we get:

$$
\begin{aligned}
P_{(i, j)(l, m)}= & \sum_{\substack{k=0\\
}}^{\infty} \mathbb{P}(Q(l, m)=k) \\
& \cdot \mathbb{1}_{0 \leq f(l)+\sum_{q=1}^{k} g\left(N^{(q)}\right)<2 d_{p}} \cdot \mathbb{1}_{N^{(k+1)}=j}
\end{aligned}
$$

As the Markov chain is irreducible and has a finite number of states, it exists a unique stationary distribution. We solve this Markov chain through a numerical method. Let us denote $\mu$ the matrix corresponding to this stationary distribution: $\mu=$ $\left(\mu_{i, j}\right)_{0 \leq(i, j) \leq K_{\max }}$. The stationary distribution $\pi$ of the subchain $\left(X_{n}\right)$ is given by $\left(0 \leq i \leq K_{\max }\right): \pi_{i}=\sum_{j=0}^{K_{\max }} \mu_{i, j}$. The mean aggregation level for the probe traffic is then computed as: MeanAgg $=\sum_{n=1}^{K_{\max }} n \cdot \pi_{n}$.

b) Second model: Cross traffic does not aggregate: By lack of place, we do not present the computation details for the second model where frame aggregation is disabled for the cross traffic. The principle is however the same. We condition by the number of successive cross traffic transmissions except that each transmission consists only in a single frame.

We now conclude by discussing our main approximations involved in our Markov model. First, for the sake of simplicity, we neglect the collisions between probe and cross traffic. Second, we do not take into account what remains at the probe traffic buffer if the number of packets exceeds $K_{\max }$. However, despite these simplifying approximations, our approach provides accurate results as discussed in the next section.

\section{NUMERICAL RESULTS}

Numerical results aim to study the behavior of the probe traffic aggregation level as a function of the network load and to validate the proposed models with regard to simulations.

\section{A. Simulation setup}

In order to assess the effectiveness of the proposed approach, we use two simulators:

- A custom-made simulator that follows the same principle as the Markov chain. It allows us to simulate more general patterns of cross traffic.

- ns-3 (version 3.30) that allows us to compare our simplified model to realistic scenarios capturing the complexity of the whole network stack.

The parameters used in the simulations and models are summarized in Table I. The network topology is composed of an AP, a station sending the probe traffic and a second one for the cross traffic. Under ns-3, we also consider a more generic scenario composed of an AP and five nodes. A node for the probe traffic and four nodes for the cross traffic. All the nodes operate on the $2.4 \mathrm{GHz}$ ISM frequency band.

\section{B. Evaluation}

Fig. 3 shows the mean aggregation level for the probe traffic when the aggregation is enabled for the cross traffic. Simulations are thus compared to model 1 of the Markov chain (Model 1). The probe packet interval varies from $50 \mu \mathrm{s}$ to $250 \mu \mathrm{s}$ and the cross traffic is set in order to have a busy time equals to $0,0.375$ and 0.625 corresponding to three load levels. Two types of cross traffic distributions are emulated in the ad-hoc simulator: exponential and deterministic.

According to these results, it appears that the model and the ad-hoc simulator follow closely the pattern of the ns-3 simulations for all the levels of cross traffic. 


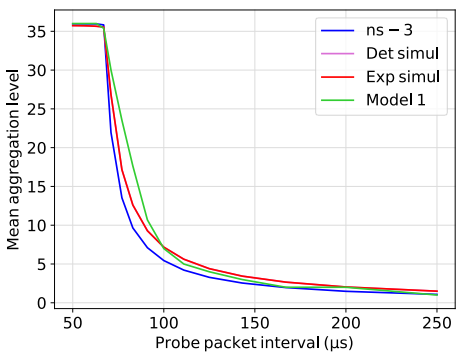

(a) 0 busy time

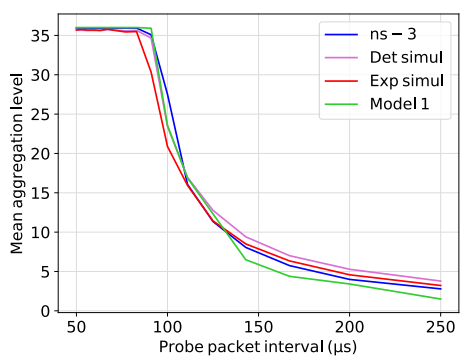

(b) 0.375 busy time

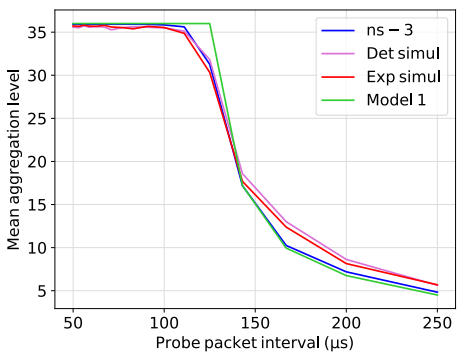

(c) 0.625 busy time

Fig. 3: Mean aggregation level versus busy time

TABLE I: 802.11n PHY and MAC Parameters.

\begin{tabular}{|c|c|}
\hline Parameter & Value \\
\hline CWmin & Minimum contention window size $=15$ \\
\hline SlotTime & Slot time $=20 \mu \mathrm{s}$ \\
\hline DIFS & Distributed inter-frame space $=50 \mu \mathrm{s}$ \\
\hline SIFS & Shortest inter-frame space $=10 \mu \mathrm{s}$ \\
\hline FCS & Frame Check Sequence $=4$ bytes \\
\hline MCS 15 & Physical transmission rate $=144.4 \mathrm{Mbps}$ \\
\hline MacHeader & MAC Header size $=34$ bytes \\
\hline PHY Overhead & PHY preamble and header time $=40 \mu \mathrm{s}$ \\
\hline Block ACK & $32 \mu \mathrm{s}$ \\
\hline Packet Size & 1024 bytes \\
\hline Channel width & $20 \mathrm{MHz}$ \\
\hline Guard Interval $(\mathrm{GI})$ & $400 \mathrm{~ns}$ \\
\hline
\end{tabular}

The obtained curves can be divided into two zones. We observe a first zone where the aggregation level is at its maximum. It corresponds to a very congested state where the probe traffic buffer is always full and exceeds the maximum number of frames that can be aggregated. When the probe packet interval increases, the aggregation decreases and follows a curve close to a hyperbola explained by the fact that the number of generated packets per second is the inverse of the probe packet interval. It can be observed in Fig. 3 that the ad-hoc simulator gives similar results for the deterministic and exponential cross traffic.

Fig. 4 provides the probe mean aggregation when frame aggregation is enabled or disabled for the cross traffic (models 1 and 2 respectively). We compare the results of the Markov chains (Model 1 and 2) to ns-3 simulations (with or without aggregation). The aggregation level is lower when cross traffic aggregation is disabled. Indeed, each cross traffic frame is sent independently, with shorter transmission times. Consequently, the probe traffic receives less packets to aggregate between two consecutive medium accesses. Also, cross traffic reaches saturation faster (as it sends less frames on average). As soon as it has always a frame to send, its buffer state does not impact the probe aggregation level. On the contrary, when cross traffic aggregates, the state of its buffer has a deeper impact on probe traffic aggregation since the cross traffic buffer state determines the transmission duration.

In the Markov chain models, the cross traffic is sent by a single queue. Fig. 5 and 6 compare ns-3 scenarios where the cross traffic is generated by one concurrent node or 4 concurrent nodes, with or without frame aggregation. The results show that the number of concurrent nodes has a negligible impact on the probe traffic aggregation level. Despite the complexity brought by the network stack layers (beacon frames, congestion, random backoff, MAC layer retries, etc.) and the different number of stations deployed in the topology, our approach is not affected in the considered scenarios.

\section{DISCUSSION}

a) Frame aggregation for load estimation: Inspired by the observation of the previous section, we discuss the feasibility to consider the mean aggregation level to estimate the channel load/busy time.

A method could consist of sending probe packet sequences with decreasing interval time from a client to a server via the wireless link with a specified data rate. Then the server estimates the mean aggregation level for the different sequences. The computation of the error between the measured aggregation levels and the theoretical ones derived from our model can then be used to infer the network load. The inferred busy time would be the one that minimizes the error between the measured and the theoretical aggregated level for a set of probe traffic intervals. Our simulation results indicate that this method should work perfectly, at least for ns-3 simulations: there are very small differences between the simulations and the models. Besides, the results with or without aggregation are significantly separated, which makes it possible to identify if the cross traffic is aggregated or not.

b) Experiments and limitations: While we shed light on the benefit of our approach based on simulations, there remains the validation of the model against testbed measurements. A part of the experiments has been already conducted. To compute the mean aggregation level, we used a sniffer that captures the frames sent through the network. The aggregation level is then computed according to the frame A-MPDU identifier and is consequently exact. This method gives results that match well with our simulations and models. However, it may be difficult to deploy it because it requires special features and configurations of the Wi-Fi card: it has to be in monitor mode, runs in privileged mode, a capture software must be installed, Wi-Fi security options must be disabled, 


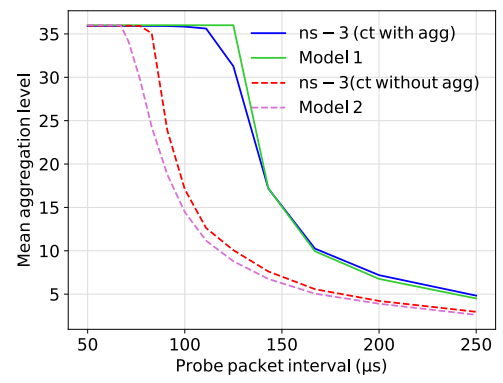

Fig. 4: Mean aggregation level for model 1 and model 2, 0.625 busy time

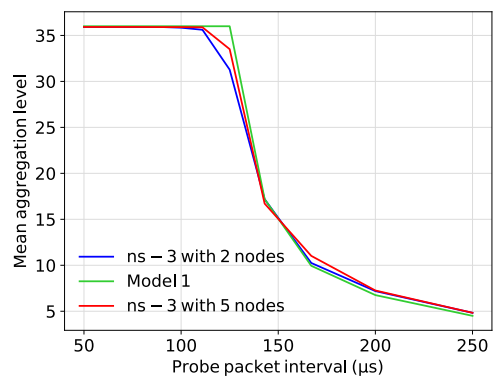

Fig. 5: Mean aggregation level for model 1 with 5 nodes, 0.625 busy time

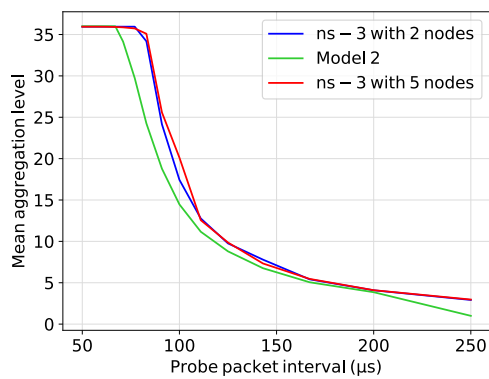

Fig. 6: Mean aggregation level for model 2 with 5 nodes, 0.375 busy time etc. A computation method implemented at the application level is thus preferable. We have tested the threshold-based method introduced in [6], [7] to estimate the aggregation level. It consists of measuring the time between the different packet reception times at the application level. If this time is below a given threshold (set as $300 \mu \mathrm{s}$ in [6] and $400 \mu \mathrm{s}$ in [7]), the two packets are considered as aggregated. Our test on Linux Ubuntu shows that this technique is inefficient due to the different times introduced by the operating system. Being able to detect frame aggregation at the application level on the server side is a technical challenge that still need to be tackled.

\section{CONCLUSION}

In this paper, we study the possibility to consider the frame aggregation level to infer the channel busy time of Wi-Fi networks. We propose two Markov chains to estimate the theoretical aggregation level for a specific scenario where a probe traffic concurrent to a cross traffic is sent to estimate the network load. It exists available bandwidth estimation tools that use the aggregation level but they are based on empirical observation and to our knowledge, there was no theoretical analysis of the aggregation level for this particular context.

We have shown through a large set of simulations performed with ns-3 and a custom-made simulator that the model allows an accurate estimation of the aggregation level. For the considered scenarios, the results show that the aggregation level could be an accurate metric to infer the network load. We are currently implementing our approach in a testbed. However, we face problems to capture the aggregation level at the application layer. An open issue is thus to propose techniques that estimate precisely the aggregation level at this layer.

\section{REFERENCES}

[1] "Cisco Visual Networking Index: Forecast and Trends, 2017-2022 White Paper".

[2] K. Sood, S. Liu, S. Yu and Y. Xiang, "Dynamic access point association using software defined networking". In 2015 International Telecommunication Networks and Applications Conference (ITNAC), 2015, pp. 226231.

[3] "Ookla Speedtest". http://www.speedtest.net/.

[4] M. Jain and C. Dovrolis, "Pathload: A measurement tool for end-to-end available bandwidth". IN Proc. of PAM, 2002, pp. 14-25.
[5] M. Li, M. Claypool, and R. Kinicki, "WBest: A Bandwidth Estimation Tool for IEEE 802.11 Wireless Networks". In LCN 2008. IEEE, 2008, pp. 374-381.

[6] A. Farshad, M. Lee, M. K. Marina, and F. Garcia, "On the impact of 802.11 frame aggregation on end-to-end available bandwidth estimation". In SECON. IEEE, Jun. 2014, pp. 108116.

[7] L. Song, and A. Striegel, "Leveraging Frame Aggregation for Estimating WiFi Available Bandwidth". In SECON 2017, March, 2017.

[8] J. Strauss, D. Katabi, and F. Kaashoek, "A measurement study of available bandwidth estimation tools'. In IMC '03, October 2003, pp. 39-44.

[9] B. S. Kim, H. Y. Hwang, and D. K. Sung, "Effect of frame aggregation on the throughput performance of IEEE 802.11n". In Proc. ACM SIGCOMM, 2008, pp. 1740-1744.

[10] Mohammad, N. and Muhammad, S and S. (2012), "Modeling and analyzing MAC frame aggregation techniques in 802.11n using bidimensional Markovian model". In Springer networked digital technologies volume 293 of the series communications in computer and information science, 2012, vol. 239, pp. 408-419.

[11] N. Hajlaoui, I. Jabri, and M. B. Jemaa, "An accurate two dimensional markov chain model for IEEE 802.11n DCF". In Wireless Networks, 2016, pp. $1-13$.

[12] A. Johnsson, B. Melander, and M. Bjorkman, "Diettopp: A first implementation and evaluation of a simplified bandwidth measurement method". In Proc. Swedish National Computer Networking Workshop (SNCNW), 2004

[13] B. Melander, M. Bjorkman and P. Gunningberg, "A new end-to-end probing and analysis method for estimating bandwidth bottlenecks". In Globecom '00. IEEE, 2000, pp. 415-420.

[14] V. Ribeiro, R. Riedi, R. Baraniuk, J. Navratil, and L. Cottrell, "pathChirp: Efficient available bandwidth estimation for network paths". In Proc. of PAM, 2003.

[15] Heung Ki Lee, Varrian Hall, Ki Hwan Yum, Kyoung Ill Kim, and Eun Jung Kim, "Bandwidth Estimation in Wireless LANs for Multimedia Streaming Services". In Adv. MultiMedia, 2007, pp. 1-9.

[16] N. Hu and P. Steenkiste, "Evaluation and characterization of available bandwidth probing techniques". In IEEE Journal on Selected Areas in Communications, Aug. 2003, vol. 21, no. 6.

[17] "IEEE Standard for Information technologyTelecommunications and information exchange between systems Local and metropolitan area networksSpecific requirements Part 11: Wireless LAN Medium Access Control (MAC) and Physical Layer (PHY) Specifications ”. IEEE Std 802.11-2016, pp. 1-3534, December 2016.

[18] "The Network Simulator ns-3". https://www.nsnam.org/.

[19] P. Ha, E. Zhang, W. Sun, F. Cui and L. Xu, "A Novel Timestamping Mechanism for Clouds and Its Application on Available Bandwidth Estimation," 2019 IEEE 39th International Conference on Distributed Computing Systems (ICDCS), Dallas, TX, USA, 2019, pp. 12-22.

[20] G. Aceto, F. Palumbo, V. Persico and A. Pescape, "Available Bandwidth vs. Achievable Throughput Measurements in 4G Mobile Networks," 2018 14th International Conference on Network and Service Management (CNSM), Rome, 2018, pp. 125-133. 